\title{
Tuberculous pancreatic and liver abscesses presented as obstructive jaundice in an immunocompetent patient
}

\author{
Eihab Subahi ${ }^{1}$, Ali Mahgoub ${ }^{1}$, Khaldun Obeidat ${ }^{2}$, Ahmed Elamin $^{1}$, and Fatima Rasoul ${ }^{1}$ \\ ${ }^{1}$ Hamad Medical Corporation \\ ${ }^{2}$ Affiliation not available
}

January 24, 2021

\begin{abstract}
We reported 24 years old Nepalese male who presented with epigastric pain and jaundiced found to have pancreatic and liver abscess suspected by abdominal CT scan and MRI, that confirmed later on by EUS-FNA with pus smear and PCR came for acid-fast bacilli. The patient responded remarkably to ATT.
\end{abstract}

\section{Introduction}

Abdominal tuberculosis (TB) commonly affects the intestinal tract, lymph nodes, peritoneum, and solid organs in varying combinations. Up to two-thirds of patients with abdominal TB have abdominal lymphadenopathy or peritoneal disease in addition to intestinal involvement. One-third may have extra-intestinal involvement also (1). Isolated hepatobiliary or pancreatic and peripancreatic TB is rare, especially when present in immunocompetent hosts. It's often occurring in the setting of miliary TB or widely disseminated disease (2). Pancreatic TB may present as a pancreatic abscess, acute or chronic pancreatitis, and cystic or solid pancreatic masses (2)(3). Clinically and radiologically pancreatic TB closely resembles a pancreatic malignancy. Therefore, most cases of pancreatic TB have been diagnosed after exploratory laparotomy surgery for suspected malignancy. However, with the use of improved imaging techniques computed tomography (CT) or more recently endoscopic ultrasound-guided fine-needle aspiration (EUS-FNA) and image-guided interventions preoperative diagnosis of pancreatic masses is now possible without going for surgery (2). EUS-guided fine-needle aspiration allows us not only to obtain tissue samples for PCR diagnosis but also to perform therapeutic aspiration of the abscess (4). Because of the non-specific clinical presentation, the disease can elude a diagnosis, but once diagnosed it responds favorably to anti-tuberculous therapies (ATT). The available literature related to hepatobiliary or pancreatic tuberculosis is mostly in the form of case reports or series. Therefore, we reported our experience with this rare form of abdominal TB, which presented with pancreatic and liver abscesses, finally diagnosed and confirmed through both radiological images and EUS-FNA.

\section{Case presentation}

24 years old Nepalese male with no past medical history presented to our emergency department with two months history of epigastric pain. the pain is dull in nature, intermittent, moderate in severity, radiating to his back, sometimes increased by food intake. After that he started to developed fever sometimes associated with chills, and yellowish discoloration of his eyes ten days before his presentation to the hospital. He also reported $10 \mathrm{Kg}$ weight loss for the last two months accompanied by loss of appetite.

On presentation, he was febrile with low-grade fever other vitals were stable. On examination, he was jaundiced, but not pale or cyanosed, with no signs of chronic liver disease. His abdomen was soft with mild 
epigastric tenderness, no hepatosplenomegaly, no palpable masses, and no flank tenderness. The examination of other systems was unremarkable. His lab on presentation showed normal leukocyte count, normal hemoglobin, and platelet count. coagulation profile was unremarkable. Urea and creatinine both were normal. his total bilirubin was high $62 \mathrm{umol} / \mathrm{L}$, mainly direct $48 \mathrm{umol} / \mathrm{L}$, alkaline phosphatase was 234 $\mathrm{U} / \mathrm{L}$, alanine transaminase (ALT) was $115 \mathrm{U} / \mathrm{L}$, aspartate transaminase was $86 \mathrm{U} / \mathrm{L}$ (AST). Albumin was low $30 \mathrm{gm} / \mathrm{L}$, amylase was high $87 \mathrm{U} / \mathrm{L}$ and lipase was normal. C-reactive protein was high $102 \mathrm{mg} / \mathrm{L}$. His interferon- $\gamma$ release assay (Quantiferon TB Gold) test was positive. HIV and hepatitis tests both were negative. blood cultures showed no growth. Tumor markers CA 19-9 and alpha fetoprotein (AFP) both were normal.

His chest x-ray was unremarkable. Initially his ultrasound abdomen showed a hetero echoic cystic lesion measuring $4.3 \times 3.9 \mathrm{~cm}$ is noted in the pancreatic head with peripheral vascularity (Figure 1a). Liver shows coarse echotexture. Mild intrahepatic biliary radical dilatation noted in the left lobe (Figure1b). No focal lesion. CBD is dilated measuring $9.9 \mathrm{~mm}$. No obvious stone could be imaged in the visualized parts of the CBD.

He underwent MRI abdomen and MRCP which showed a relatively well-defined lobulated heterogenous T2 hyperintense necrotic lesion measuring $3 \times 5.2 \times 5.6 \mathrm{~cm}$ is noted involving the pancreatic head and the caudate lobe of the liver, displacing the adjacent structures. It shows peripheral thin enhancing and $\mathrm{T} 2$ hypointense rim and some internal enhancing septations with diffusion restriction of its central fluid contents and wall is also noted. This most likely represents a large abdominal cold abscess arising from a conglomerate necrotic peripancreatic / portacaval lymph nodal mass which has further extended to the pancreatic head and the caudate lobe of the liver. Two small adjacent peripherally enhancing cystic lesions in the segment VIII of the liver are noted which appear bright on the DWI, likely representing microabscesses. The necrotic abscess is compressing the CBD and causing moderate upstream CBD (about $13 \mathrm{~mm}$ ) and mild intrahepatic duct dilatation. The radiologist suggested considering Tuberculosis infection as a first diagnosis.

CT abdomen showed Redemonstrations of lesion that was seen in MRI images which involving mainly the hepatic caudate lobe extending to porta hepatis, peripancreatic and pancreatic head, suggestive of abscess (tubercouls), with other adjacent necrotic lymph nodes in the upper abdomen. Tiny hepatic lesions in segment VIII likely microabscesses, and inflammatory changes in segment VI. Mass effect on the CBD (with upstream dilatation) and portal vein (Figure 2). CT chest was unremarkable.

He underwent Endoscopic ultrasound (EUS) with upper GI endoscopy for aspiration of the abscess and definitive diagnosis. EUS showed Intrahepatic bile ducts were dilated. There were multiple large lymph nodes with anechoic areas inside (necrotic LNs) in the liver hilum, para-aortic, para-duodenal areas.

Pancreatic parenchyma had diffused stranding and hyperechogenic foci. A cystic mass lesion measuring 40 x $40 \mathrm{~mm}$ was seen between the liver hilum and pancreatic head. The cystic mass had internal echogenicity, abutting liver parenchyma and pancreatic head, and was compatible with an abscess. CBD was normal but it was not possible to follow CBD due to the compression of the cystic mass. CHD was slightly dilated. A fine-needle aspiration from the

abscess was performed using a 19G FNA needle. Approximately $20 \mathrm{ml}$ of grey-yellowish colored pus was aspirated from the abscess. TB culture, TB PCR, AFP stain, cytopathology and bacterial culture was sent. Later on, his AFP smear and PCR came positive for acid-fast bacilli (Mycobacterium tuberculosis), and the fluid culture showed Scanty growth Stenotrophomonas maltophilia treated with trimethoprim-sulfamethoxazole for 7 days. The diagnosis of pancreatic and liver tuberculous abscesses was made based on the above findings and results, then subsequently the patient was treated with anti-tuberculous therapy (ATT) which include Isoniazide, Rifampicin, Ethambutol, and Pyrazinamide. He showed a good clinical response to the ATT.

\section{Discussion}

Tuberculosis (TB) is a multisystemic infectious disease caused by various strains of mycobacteria, usually Mycobacterium tuberculosis. Although pulmonary TB is the most common presentation of disease; 
extrapulmonary TB (EPTB) accounts for nearly 20 percent of all cases of TB in immunocompetent hosts, and Tuberculosis (TB) of the abdomen is seen in $12 \%$ of patients with miliary TB (5)(6).By definition, EPTB describes the occurrence of TB at sites other than the lung. EPTB can occur in almost any organ system, with the most common sites of infection being the lymph nodes, pleura, genitourinary system, and bone (5)(7). Abdominal TB is the sixth most common site for EPTB, and includes infection anywhere in the gastrointestinal tract, peritoneum, and intra-abdominal organs such as the spleen, liver, and pancreas (7).

The clinical presentation of abdominal TB especially pancreatic and hepatobiliary involvement is often insidious, with nonspecific constitutional symptoms occurring frequently (5). In a study by Saluja et al. , the three most common presenting complaints in patients found to have pancreatic TB were abdominal pain, jaundice, and weight loss (8). Individuals infected with pancreatic TB may also present with fever, gastrointestinal hemorrhage secondary to splenic vein thrombosis, and anorexia (8). If pancreatic TB is suspected, preliminary testing such as tuberculin skin testing and an interferon- $\gamma$ release assay for TB may be negative in patients. Sharma et al. suggest that the sensitivity of tuberculin skin testing in patients with abdominal tuberculosis may range from 58 to 100 percent (5). With the wide-ranging sensitivities of TB screening modalities and an often nonspecific and varied clinical presentation of pancreatic and hepatobiliary $\mathrm{TB}$, diagnosis of infection relies heavily on radiologic and histopathologic findings. In our case, the patient presented with abdominal pain mainly epigastric associated with jaundice and weight loss. His interferon- $\gamma$ release assay for TB (Quantiferon TB Gold) was positive from the beginning.

Ultrasonography or computed tomography (CT) are often first-line diagnostic modalities in patients presenting with signs of pancreatic pathology $(2)(5)$. Ultrasound is often the first investigation used for diagnosis of pancreatic tuberculosis which may reveal a focal hypoechoic mass or cystic lesion of the pancreas mostly situated in the head and uncinate process of the pancreas (2).

CT scan is still regarded as the investigation of choice for pancreatic pathology (9).CT scan may show hypodense lesion with irregular border in the head of the pancreas, diffuse enlargement of the pancreas, or enlarged peripancreatic lymph nodes (9). The presence of hypodense peripancreatic lymph nodes with rim enhancement, ascites, and/or mural thickening affecting the ileo-caecal region suggests the pancreatic tuberculosis (9). Magnetic resonance imaging (MRI) findings of focal pancreatic tuberculosis include a sharply delineated mass in the pancreatic head showing heterogeneous enhancement which is hypointense on fat-suppressed T1-weighted images and shows a mixture of hypo- and hyperintensity on T2-weighted images (10). In our case, his US showed a hetero echoic cystic lesion in the pancreatic head, and the CT showed Pancreatic parenchyma had diffused stranding and hyperechogenic foci, lastly his MRI showed a relatively well-defined lobulated heterogeneous T2 hyperintense necrotic lesion involving the pancreatic head and the caudate lobe of the liver. Techniques for pancreatic biopsy include CT or ultrasound-guided percutaneous biopsy, surgical biopsy, or endoscopic ultrasound (EUS) guided fine-needle aspiration (FNA)(2). The American Joint Commission on Cancer (AJCC) recommends EUS-FNA as the diagnostic modality of choice in patients with pancreatic masses and has found it to be the most sensitive and specific method for identifying the etiology of pancreatic masses (2). The presence of on-site cytology is imperative in the diagnosis of pancreatic TB, as the immediate interpretation of the specimen will allow clinicians to request appropriate cultures (2). Acid-fast bacilli are commonly not seen with FNA. In a study by Farar et al., nearly 40 percent of patients with abdominal TB had staining that was negative for acid-fast bacilli (11). Clinicians should be cognizant of the relatively low yield of FNA specimens to reveal acid-fast bacilli and thus culture the specimen for evidence of Mycobacterium tuberculosis (11).In our experience, the patient underwent EUS-FNA to confirm the diagnosis and for sample collection, his AFP smear and PCR came positive for acid-fast bacilli (Mycobacterium tuberculosis) .

Once the diagnosis of abdominal TB has been made, standard anti-TB therapy appears to be successful in the management of this infection. A minimum of 6 months of anti-TB therapy is often indicated to achieve resolution of pancreatic lesions and alleviation of symptoms. Follow-up CT imaging after treatment may reveal the complete resolution of pancreatic lesions secondary to tuberculosis and may guide clinicians 
regarding the duration of therapy (12).

\section{Conclusion}

In conclusion, hepatobiliary, peripancreatic, and pancreatic tuberculous abscesses are extremely rare. As the clinical presentation is often fugue and radiographic pictures may mimic the malignancy, the clinicians should have a high index of suspicion of tuberculosis, especially in young patients from areas where the infection is endemic. application of endoscopic ultrasound-guided fine-needle aspiration (EUS-FNA) to obtain pathological evidence are extremely important to a correct diagnosis. Provided diagnosis is established, anti-tuberculous therapy may be sufficient to the alleviation of the symptoms and possible resolution of the abscess.

\section{Acknowledgment}

The authors would like to acknowledge the internal medicine residency program and the Qatar National Library for their support.

\section{Statement of Ethics}

The patient consented to the publication of his case.

\section{Disclosure statement}

The authors have no conflict of interest.

\section{Funding sources}

This work was funded by the Qatar National Library.

\section{Authors contributions}

Dr. Eihab A. Subahi, Dr. Ali B. Mahgoub, Dr. Khaldun Obeidat, and Dr. Ahmed M. Elamin all wrote the manuscript. Dr. Fatima A. Rasoul reviewed and edited

the manuscript.

\section{Data availability statement}

The data that support the findings of this study are available from the corresponding author upon reasonable request.

\section{References}

1. Leder RA, Low VHS. Tuberculosis of the abdomen. Vol. 33, Radiologic Clinics of North America. 1995. p. 691-705.

2. Kaushik N, Schoedel K, McGrath K. Isolated pancreatic tuberculosis diagnosed by endoscopic ultrasoundguided fine needle aspiration: A case report. J Pancreas . 2006 Mar 9 [cited 2020 Nov 15];7(2):205-10.

3. Bakhshi G, Bhattu A. Primary Pancreatic Tuberculosis: A Case Report . [cited 2020 Nov 15].

4. Song TJ, Lee SS, Park DH, Lee TY, Lee SO, Seo DW, et al. Yield of EUS-guided FNA on the diagnosis of pancreatic/peripancreatic tuberculosis. Gastrointest Endosc . 2009 Mar 1 [cited 2020 Nov 15];69(3):484-91.

5. S K Sharma 1 AM. Extrapulmonary tuberculosis - PubMed. Indian J Med Res . 2004 [cited 2020 Nov $15] ; 120(4): 316-53$.

6. Haddad FS, Ghossain A, Sawaya E, Nelson AR. Abdominal tuberculosis. Dis Colon Rectum . 1987 Sep [cited 2020 Nov 15];30(9):724-35.

7. M P Sharma 1 VB 2. Abdominal Tuberculosis - PubMed. Indian J Med Res . 2004 [cited 2020 Nov 15];120(4):305-15. 
8. Saluja SS, Ray S, Pal S, Kukeraja M, Srivastava DN, Sahni P, et al. Hepatobiliary and pancreatic tuberculosis: A two decade experience. BMC Surg . 2007 Dec 24 [cited 2020 Nov 15];7(1):10.

9. D Takhtani 1, S Gupta, K Suman, N Kakkar, S Challa, J D Wig SS. Radiology of pancreatic tuberculosis: a report of three cases - PubMed. Am J Gastroenterol . 1996 [cited 2020 Nov 15];91(9):1832-4.

10. De Backer AI, Mortelé KJ, Bomans P, De Keulenaer BL, Vanschoubroeck IJ, Kockx MM. Tuberculosis of the Pancreas: MRI Features. Am J Roentgenol . 2005 Jan 23 [cited 2020 Nov 15];184(1):50-4.

11. FARER LS, LOWELL AM, MEADOR MP. EXTRAPULMONARY TUBERCULOSIS IN THE UNITED STATES1. Am J Epidemiol . 1979 Feb 1 [cited 2020 Nov 15];109(2):205-17.

12. G Brusko 1, W S Melvin, J J Fromkes ECE. Pancreatic tuberculosis - PubMed. Am Surg . 1995 [cited 2020 Nov 15];61(6):513-5.

\section{List of figures}

\section{Figure number Comment (description)}

Figure 1

Selected axial ultrasound images of the upper abdomen. (a) A heterogeneously echoic lesion with multi

Figure 2

Axial images of contrast enhanced computed tomography of the abdomen. Well defined lobulated hypo

\section{Hosted file}

image1.emf available at https://authorea.com/users/356525/articles/505531-tuberculouspancreatic-and-liver-abscesses-presented-as-obstructive-jaundice-in-an-immunocompetentpatient

\section{Hosted file}

image2.emf available at https://authorea.com/users/356525/articles/505531-tuberculouspancreatic-and-liver-abscesses-presented-as-obstructive-jaundice-in-an-immunocompetentpatient 